\title{
REVIEW
}

\section{Chemosaturation Percutaneous Hepatic Perfusion: A Systematic Review}

\author{
Arndt Vogel · Sanjay Gupta · Martin Zeile · Rebecca von Haken · Roland Brüning · \\ Gösta Lotz · Alexander Vahrmeijer · Thomas Vogl $\cdot$ Frank Wacker
}

Received: July 19, 2016 / Published online: October 31, 2016

(C) The Author(s) 2016. This article is published with open access at Springerlink.com

\begin{abstract}
The Hepatic CHEMOSAT ${ }^{\circledR}$ Delivery System is an innovative medical device for the treatment of patients with unresectable primary liver tumors or unresectable hepatic metastases from solid organ malignancies. This system is used to perform chemosaturation percutaneous hepatic perfusion (CS-PHP), a procedure in which a high dose of the chemotherapeutic agent melphalan is delivered directly to the
\end{abstract}

Enhanced content To view enhanced content for this article go to http://www.medengine.com/Redeem/ FC07F0602F4362E4.

\footnotetext{
A. Vogel $(\bowtie)$

Department of Gastroenterology, Hepatology and Endocrinology, Medizinische Hochschule

Hannover, Hannover, Germany

e-mail: vogel.arndt@mh-hannover.de

S. Gupta

Department of Anaesthesiology, University Hospital Southampton, Tremona Road, Southampton,

Hampshire, UK

M. Zeile · R. Brüning

Department of Radiology and Neuroradiology,

Asklepios Clinic Hamburg-Barmbek, Hamburg,

Germany

R. von Haken

Department of Anaesthesiology, University Clinic

Heidelberg, Heidelberg, Germany
}

liver while limiting systemic exposure. In a clinical trial program, CS-PHP with melphalan significantly improved hepatic progression-free survival in patients with unresectable hepatic metastases from ocular or cutaneous melanoma. Clinically meaningful hepatic responses were also observed in patients with hepatocellular carcinoma or neuroendocrine tumors. Furthermore, the results of published studies and case reports demonstrated that CS-PHP with melphalan resulted in favorable tumor response rates in a range of tumor histologies (ocular or cutaneous melanoma, colorectal cancer, and hepatobiliary tumors). Analyses of

G. Lotz

Department of Anaesthesiology, University Clinic Frankfurt, Frankfurt am Main, Germany

A. Vahrmeijer Department of Surgery, Leiden University Medical Centre, Leiden, The Netherlands

T. Vogl Institute of Diagnostic and Interventional Radiology, University Clinic Frankfurt, Frankfurt am Main, Germany

F. Wacker Institute of Diagnostic and Interventional Radiology, Medizinische Hochschule Hannover, Hannover, Germany 
the safety profile of CS-PHP revealed that the most common adverse effects were hematologic events (thrombocytopenia, anemia, and neutropenia), which were clinically manageable. Taken together, these findings indicate that CS-PHP is a promising locoregional therapy for patients with primary and secondary liver tumors and has a acceptable safety profile.

Funding: Delcath Systems Inc., New York, NY, USA.

Keywords: Chemosaturation percutaneous hepatic perfusion; Hepatic metastases; Melphalan; Oncology; Primary liver tumors

\section{INTRODUCTION}

In an effort to control disease, palliate symptoms, and extend survival, several types of treatment approaches have been pursued in patients with primary liver tumors or hepatic metastases [1-18]. The Hepatic CHEMOSAT ${ }^{\circledR}$ Delivery System (Delcath Systems Inc., New York, NY, USA) is an innovative medical device for the treatment of patients with unresectable primary liver tumors or unresectable hepatic metastases from solid organ malignancies, in which a high dose of the chemotherapeutic agent melphalan is delivered directly to the liver while limiting systemic exposure. The concept of the procedure was developed several decades ago, but is so far not established in daily clinical practice [19]. The Hepatic CHEMOSAT ${ }^{\circledR}$ Delivery System received CE mark approval in the European Union (EU) on April 13, 2011, and the product was launched commercially in February 2012.

Promising candidates for local ablative therapies are either patients with primary liver cancers, such as hepatocellular carcinoma and cholangiocellular carcinoma, or patients with extrahepatic cancers and liver-limited disease. Hepatocellular carcinoma (HCC) is one of the most lethal and prevalent cancers worldwide. In 2008 , there were 748,300 new cases of HCC and 695,900 deaths registered [20]. HCC is commonly caused by hepatitis $\mathrm{B}$ and $\mathrm{C}$ infections $(\sim 75 \%)$, chronic exposure to toxins such as aflatoxin B, non-alcoholic and alcohol steatohepatitis, or less frequently with hereditary liver diseases. The prognosis of patients with HCC is dismal and the mortality rates are almost the same as the incidence rates. Potentially curative treatments for patients with early stage disease are liver transplantation (LT), resection, and radiofrequency or microwave ablation (RFA/MWA) [21]. Transarterial chemoembolization (TACE) is the recommended treatment for patients with intermediate stage HCC on the basis of two prospective trials with highly selected patients and one systematic review and meta-analysis $[22,23]$. HCC is typically resistant to systemic chemotherapy and the multi-kinase inhibitor sorafenib is the only approved agent for patients with advanced disease.

Intrahepatic cholangiocarcinoma (ICC), the second most common primary liver tumor, involves a heterogeneous group of malignancies affecting the biliary epithelium [24]. Radical resection is the only curative treatment option. In cases with a potentially curative surgery, however, 5-year survival rates of only $25-30 \%$ are reported indicating the unmet need for multimodal treatment strategies to improve the cure rate of patients with cholangiocarcinoma [25]. In metastatic disease, chemotherapy improves quality of life and survival, and gemcitabine with cisplatin represents the standard of care on the basis of recently published phase II and III clinical trials 
[26]. However, all patients ultimately progress on this therapy, so clinical trials of new and better agents and innovative treatment strategies are essential to expand the existing treatment options for patients with cholangiocarcinoma.

Metastases to the liver are more common than primary liver tumors [27]. The liver is the dominant site of metastatic disease for patients with a wide variety of primary tumor types, including ocular melanoma, colorectal carcinoma (CRC), and neuroendocrine tumors (NETs) [28-31]. More rarely, hepatic metastases also arise as the sole site of metastasis in a subgroup of patients with soft tissue sarcomas, cutaneous melanoma, breast cancer, ovarian cancer, thyroid cancer, non-small cell lung cancer, or renal cell cancer. Local and locoregional therapies have been shown to be well tolerated and can contribute to tumor control in the context of comprehensive oncologic treatment strategies, and may prolong survival of patients with liver limited metastasis. Unfortunately, however, only a few high-quality clinical trials are available and randomized prospective clinical trials enrolling larger numbers of patients need to be carried out to elucidate the precise value of these treatments in combination with systemic chemotherapy.

The objective of this review is to provide a comprehensive framework to guide clinical practice in the use of this procedure and the sharing of clinical experience across multiple disciplines, including interventional radiology, anesthesiology, and medical and surgical oncology. The results of clinical trials of CS-PHP, as well as retrospective studies and case reports, are also described. Multiple discussions, including an investigator meeting in Paris, among European experts in the field of CS-PHP formed the basis of this review.

\section{Compliance with Ethics Guidelines}

This article does not contain any new studies with human or animal subjects performed by any of the authors.

\section{CS-PHP PROCEDURE}

\section{Rationale}

Delcath Systems has developed a drug/device combination product as a minimally invasive alternative to open-surgical isolated hepatic perfusion (IHP). The product is composed of the chemotherapeutic agent melphalan hydrochloride and a number of sterile, single-use components, including catheters and an extracorporeal circuit with hemofiltration cartridges. The Delcath device is used in a procedure known as CS-PHP to percutaneously deliver a high dose of melphalan to the liver via the hepatic artery, while minimizing systemic exposure to the drug by filtering blood before it is returned to the systemic circulation.

\section{Chemotherapeutic Agent}

Melphalan was selected as the chemotherapeutic agent for use with CS-PHP because it showed both efficacy and reversible hepatic toxicity in the treatment of hepatic metastases from a variety of tumors, including melanoma, CRC, HCC, and NETs in the analogous regional procedure of IHP [32-36]. The principal toxicity of melphalan is bone marrow suppression, including thrombocytopenia, anemia, and neutropenia.

\section{Technique}

The Hepatic CHEMOSAT ${ }^{\circledR}$ Delivery System is used to conduct CS-PHP. A schematic overview 
of how the components of the CHEMOSAT ${ }^{\circledR}$ Delivery System work together is provided in Fig. 1.

The CS-PHP procedure is conducted in an interventional radiology suite with the patient under general anesthesia. Induction of anesthesia, intubation, and choice of anesthetic and muscle relaxant are left to the discretion of the anesthesiologist. Both central venous and invasive lines are required, and arterial pressure must be continuously monitored. Heart rate, temperature, and acid-base profile are also measured throughout the procedure, which takes approximately 3-4 h to complete.
In CS-PHP, melphalan is delivered directly into the hepatic artery via a catheter in the proper hepatic artery. Prior to placement of the catheter an angiogram of the celiac trunk and the superior mesenteric artery is performed, the latter with acquisition of a portal venous phase. A double-balloon catheter, usually advanced through the right femoral vein, is positioned in the inferior vena cava (IVC) with the cranial balloon in the right atrium-IVC junction and the caudal balloon in the infrahepatic IVC above the renal veins to isolate and collect hepatic venous outflow.

An extracorporeal circuit is established in a stepwise fashion. First, a closed loop between

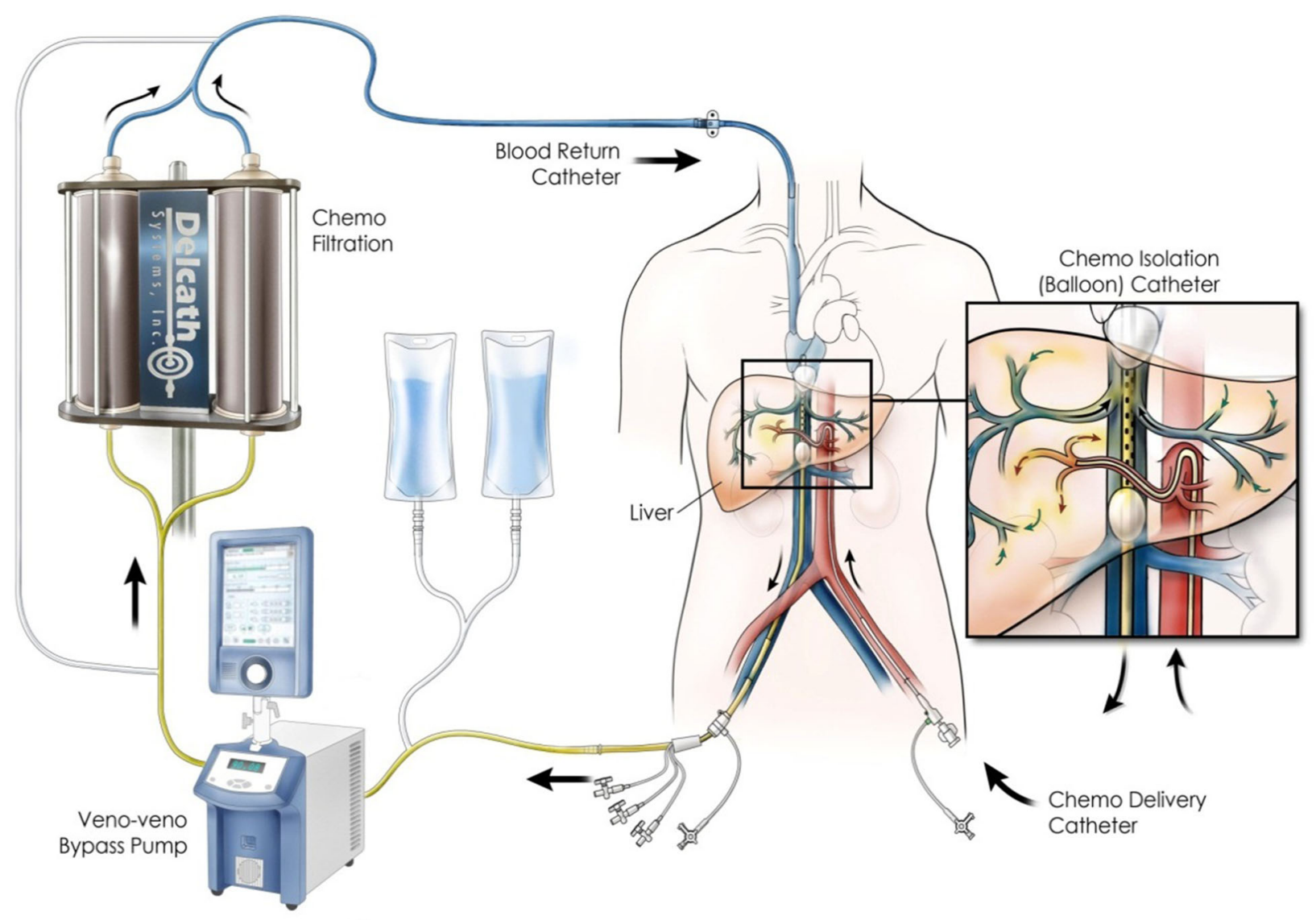

Fig. 1 Overview of CS-PHP treatment. Reproduced with permission from Delcath 
the double-balloon catheter and jugular sheath is created with a centrifugal pump to maintain flow. The cephalad balloon is filled while still in the atrium with approximately $20 \mathrm{~mL}$ saline and pulled back. Once this balloon is in position, the lower balloon is inflated. Contrast injection is used to exclude leakage and check for retrograde filling of the hepatic veins. Blood filtration through two activated carbon filter cartridges arranged in parallel is started. Once the extracorporeal circuit is established and the patient and extracorporeal blood flow are stable, melphalan is administered (typically as $100-\mathrm{mL}$ aliquots of diluted melphalan in $500 \mathrm{~mL}$ ) via the catheter positioned in the hepatic artery. After each aliquot, digital scan angiography is performed to check for patency and anterograde flow. In cases of vasospasm, intra-arterial injection of vasodilators is recommended. Hepatic venous outflow is sent through an extracorporeal filtration system to lower the concentration of melphalan in the blood before being returned to the systemic circulation via an internal jugular vein sheath. Extracorporeal filtration continues for an additional $30 \mathrm{~min}$ after termination of the melphalan infusion to filter any drug released from the liver; filtration efficiency is $93 \%$.

\section{Procedure Team}

The performance of the CS-PHP procedure requires a multidisciplinary procedural team with the knowledge and skills required to care for patients undergoing this procedure. An interventional radiologist leads the entire team during the procedure through communication and coordination. A surgical or medical oncologist is responsible for the complete management of the patient from prior to the procedure through follow-up. An anesthesiologist is responsible for general anesthesia, coagulation management, and postoperative care. A perfusionist establishes, monitors, and controls the extracorporeal circuit. A certified healthcare provider for chemotherapy delivery (not required in all countries) is responsible for melphalan administration. The interventional radiology staff assists in the procedure and imaging. A pharmacist is responsible for melphalan preparation.

\section{Patient Selection}

Careful selection of appropriate candidates for CS-PHP is critical for a successful outcome. Patients must have either surgically unresectable primary or metastatic cancer of the liver. Other evidence-based, local-regional or systemic treatment options need to be discussed in a multidisciplinary tumorboard. Magnetic resonance or computed tomography imaging of the liver must be completed to document the position of liver metastases and evaluate adjacent vascular structures and the degree of vascularity. A full-body computed tomography scan should be conducted to determine whether there is extrahepatic spread. Patients with a tumor burden exceeding $50-60 \%$ should not be treated outside of clinical trails, because there is currently not sufficient evidence for the long-term benefit and the potentially increased side effect profile. Patients should not be treated if they have advanced liver cirrhosis with portal hypertension or encephalopathy. Patients must have adequate hepatic function (Child-Pugh A; total bilirubin $<3.0 \mathrm{mg} / \mathrm{dL}$; aspartate aminotransferase and alanine aminotransferase $<5$ times the upper limit of normal; adequate hematologic function (hemoglobin >9 g/dL; absolute neutrophil 
count $>1.3$ cells $/ \mu \mathrm{L}$; platelets $>80$ thousand per microliter); and adequate renal function (serum creatinine $<1.5 \mathrm{mg} / \mathrm{dL}$, unless measured creatinine clearance is $>60 \mathrm{~mL} / \mathrm{min} / 1.73 \mathrm{~m}^{2}$ ). Patients must also have an Eastern Cooperative Oncology Group (ECOG) score of 0 or 1 before CS-PHP.

CS-PHP should not be performed in patients with any of the following:

- Childs B or C cirrhosis or evidence of portal hypertension by endoscopy or radiologic imaging

- A history of transient ischemic attacks

- Heart failure, with a left ventricular ejection fraction $<50 \%$

- Significant chronic obstructive pulmonary disorder or other chronic pulmonary restrictive disease that would make the patient ineligible for general anesthesia

- Body weight $<35 \mathrm{~kg}$ (because of anatomical and physical limitations with the size of the double-balloon catheter)

- Severe allergic reactions to iodinated contrast that cannot be controlled by antihistamines and steroids

- Prior hypersensitivity reaction to melphalan, a documented latex allergy, or a history of hypersensitivity to heparin or presence of heparin-induced thrombocytopenia antibodies

- A history of bleeding disorders or evidence of an intracranial abnormality that puts the patient at risk for bleeding with anticoagulation

- A history of gastrinoma

\section{Patient Preparation}

Angiographic mapping of the hepatic arterial circulation with embolization of the gastroduodenal artery and certain branches supplying the pancreas, stomach, or duodenum is recommended to avoid inadvertent reflux of melphalan into gastrointestinal arterial branches and gastrointestinal toxicity. Completion is recommended at least 1 week prior to the CS-PHP procedure. In patients with a long proper hepatic artery allowing for secure positioning of the delivery catheter, gastroduodenal artery embolization can be omitted.

Accurate clinical assessment of comorbidities, particularly exclusion of ischemic heart disease, is necessary. The patient is admitted to the hospital the night before for preparation for the CS-PHP procedure. Intravenous (IV) hydration is started to ensure an adequate fluid preload before the procedure; however, this is not consistently done at all institutions, as some centers believe that a fluid preload increases the risk of edema, particularly of the neck, airways, and pulmonary interstitium. Patients on angiotensin-converting enzyme inhibitors should discontinue such medications 2 days before the procedure, whereas those on other antihypertensive medications should discontinue these medications on the day of the procedure. At the discretion of the procedural team, patients on beta-blockers may have their dose halved before the procedure.

Prior to CS-PHP, proton pump inhibitors are administered to prevent gastritis, which could occur as a result of regional melphalan absorption during the procedure. Patients with a history of hepatobiliary surgery or ablative procedures are given antibiotics prophylactically to prevent infections.

\section{Support During and After CS-PHP}

Heparin is administered by the anesthesiologist to maintain activated clotting time at therapeutic levels. Heparin is administered at the direction of the interventional radiologist 
before he/she isolates the liver and prior to the initiation of the extracorporeal circuit by the perfusionist. Vital signs are monitored continuously throughout the procedure by the anesthesiologist.

All patients will experience hypotension at two points during the procedure: when the venous balloons are inflated and when the filters in the bypass circuit are activated [37]. The blood pressure decrease is managed with prehydration, intraoperative fluid boluses, and IV vasopressors until blood pressure normalizes. Vasopressors are administered by the anesthesiologist to maintain a mean arterial pressure above $65 \mathrm{mmHg}$ to prevent ischemic injury to the heart and brain. The mechanism of hypotension during the filtration phase has not been clearly elucidated. It was previously speculated that hypotension was due to sequestration of catecholamines; however, some CS-PHP providers believe this is unlikely since patients always respond with sympathetic-mediated tachycardia. Other CS-PHP providers believe hypotension is likely a systemic inflammatory response with associated cytokine release or is induced by nitric oxide. In some institutions, remifentanyl is not administered for CS-PHP because of its profound sympathetic suppression, which makes the hypotensive phase more challenging. Vasopressor support may be weaned during the 30-min melphalan infusion and is not required after conclusion of the procedure.

Arterial patency is assessed by the interventional radiologist several times during the procedure by injection of contrast media into the hepatic artery catheter to ensure that there is no vasospasm of the hepatic artery that could result in melphalan reflux into proximal gastrointestinal branches. If hepatic spasm is seen, nitroglycerin is administered by the interventional radiologist via intra-arterial injection. The procedure is terminated by the interventional radiologist if the spasm does not resolve with nitroglycerin administration.

After CS-PHP, anticoagulation is reversed according to the institutional standard of care. The vascular access sheaths are removed when coagulation is normal; a vascular occlusion device might be used to seal the arterial puncture. Immediately following completion of the procedure, patients are monitored for $6-16 \mathrm{~h}$ at an intermediate care unit or ICU and then on a general ward for 2-3 days. Early mobilization and early intestinal motility (hence oral intake) have been correlated in some institutions with improved discharge times to the general ward. Patients must be periodically monitored for hematologic toxicity following the procedure for up to 3 weeks and may require growth factor support or packed red blood cell or platelet transfusions.

\section{EVIDENCE FOR EFFECTIVENESS OF CS-PHP}

\section{Clinical Trials}

The use of CS-PHP with melphalan was tested in a formal clinical trial program that included the following studies:

- Phase 1, single-center [US National Cancer Institute (NCI)], sequential, dose-escalation study to determine the maximum tolerated dose (MTD) of CS-PHP with melphalan in patients with unresectable hepatic metastases from cutaneous or ocular melanoma or other tumor types [38].

- Phase 2, open-label, single-center (NCI), nonrandomized, uncontrolled study to examine the efficacy of CS-PHP with melphalan in patients with unresectable primary hepatic malignancies (i.e., HCC or ICC) or unresectable metastatic 
hepatic malignancies from other tumor types [gastrointestinal adenocarcinoma (primarily CRC), NET, and cutaneous or ocular melanoma] [39].

- Phase 3, randomized, controlled, multicenter study (11 active sites in the USA) to evaluate the efficacy, safety, and tolerability of CS-PHP with melphalan compared with best alternative care (BAC) in patients with unresectable hepatic metastases from cutaneous or ocular melanoma [39]. Patients in the BAC group were allowed to cross over to CS-PHP at the time of documented hepatic progression, provided they continued to meet eligibility criteria for the study at the time of crossover. The primary endpoint for this study was hepatic progression-free survival (hPFS); secondary endpoints were hepatic objective response (hOR) and overall survival (OS).

In the phase 1 study, response was evaluable in 27 patients (Table 1) [38]. Of 10 patients with ocular melanoma, 50\% had an objective tumor response and two had a complete response at 10 and 12 months, respectively. Additionally, two of four patients with hepatic metastases from
NET had ongoing partial responses at 5 and 7 months. Dose-limiting toxicities (DLTs), as determined by the investigators, are summarized in Table 2. The MTD of melphalan delivered by CS-PHP was determined to be $3.0 \mathrm{mg} / \mathrm{kg}$ since only one patient had a DLT at this dose. All DLTs were events related to bone marrow suppression, including neutropenia, febrile neutropenia, leukopenia, and thrombocytopenia.

In the phase 2 study (Table 3), objective responses were seen in $75 \%$ of ocular melanoma patients, $12.5 \%$ of hepatocellular carcinoma patients, and $41.7 \%$ of NET patients [39]. In the last of these cohorts, response varied by type of NET. The hOR rate was $30.8 \%$ in patients with pancreatic NET, $66.7 \%$ in those with carcinoid NET, and $50.0 \%$ in those with other types of NET. All responses were partial responses. An additional $46.2 \%$ of patients within the pancreatic NET cohort had stable disease. The most common toxicities were related to bone marrow suppression, the known principal toxicity of melphalan, including thrombocytopenia, anemia, and neutropenia. Most of these events were not associated with clinical sequelae.

Table 1 Treatment responses in phase 1 study of CS-PHP [38]

\begin{tabular}{|c|c|c|c|c|c|c|c|}
\hline \multirow[t]{2}{*}{ Tumor type } & \multirow[t]{2}{*}{ Number } & \multicolumn{2}{|c|}{ Partial response } & \multicolumn{2}{|c|}{ Complete response } & \multicolumn{2}{|c|}{ Overall response } \\
\hline & & $\bar{n}$ & Duration (months) & $\bar{n}$ & Duration (months) & $n$ & $\%$ \\
\hline Ocular melanoma & 10 & 3 & $7,9+, 11+$ & 2 & 10,12 & 5 & 50 \\
\hline Cutaneous melanoma & 2 & - & - & - & - & 0 & - \\
\hline Neuroendocrine & 3 & 2 & $3+, 7+$ & - & - & 2 & - \\
\hline Colorectal & 1 & - & - & - & - & 0 & - \\
\hline Adrenal & 1 & 1 & $10+$ & - & - & 1 & - \\
\hline Other & 7 & - & - & - & - & 0 & - \\
\hline Total & $27^{*}$ & 6 & - & 2 & - & 8 & 29.6 \\
\hline
\end{tabular}

* Of the 27 patients that were assessed for hepatic response only the results of 24 are provided 
Table 2 DLTs in phase 1 study [38]

\begin{tabular}{lllll}
\hline \multicolumn{4}{l}{ Melphalan dose $(\mathbf{m g} / \mathbf{k g})$} & \\
\cline { 2 - 5 } & $\mathbf{2 . 0}(\boldsymbol{n}=\mathbf{1 4})$ & $\mathbf{2 . 5}(\boldsymbol{n}=\mathbf{3})$ & $\mathbf{3 . 0}(\boldsymbol{n}=\mathbf{1 0})$ & $\mathbf{3 . 5}(\boldsymbol{n}=\mathbf{6})$ \\
\hline No. of patients with a DLT & 0 & 0 & 1 & 2 \\
Decreased neutrophil count & 0 & 0 & - & 2 \\
Decreased white blood cell count & 0 & 0 & 1 & 1 \\
Decrease platelet count & 0 & 0 & 1 & 2 \\
Febrile neutropenia & 0 & 0 & 1 & 1 \\
\hline
\end{tabular}

$D L T$ dose-limiting toxicity

Table 3 Hepatic responses by RECIST in phase 2 study [39]

\begin{tabular}{lllll}
\hline $\begin{array}{l}\text { Best } \\
\text { response }\end{array}$ & $\begin{array}{l}\text { Neuroendocrine } \\
\text { tumor }(\boldsymbol{n}=\mathbf{2 4})\end{array}$ & $\begin{array}{l}\text { Primary hepatic } \\
\text { cancer }(\boldsymbol{n}=\mathbf{8})\end{array}$ & $\begin{array}{l}\text { Ocular melanoma } \\
(\boldsymbol{n}=\mathbf{4})\end{array}$ & $\begin{array}{l}\text { Adenocarcinoma } \\
(\boldsymbol{n}=\mathbf{1 7})^{\mathbf{a}}\end{array}$ \\
\hline $\mathrm{CR}$ & 0 & 0 & 0 & 0 \\
$\mathrm{PR}$ & 10 & 1 & 3 & 0 \\
$\mathrm{SD}$ & 6 & 4 & 1 & 4 \\
$\mathrm{PD}$ & 3 & 1 & 0 & 5 \\
Not available & 5 & 2 & 0 & 8 \\
\hline
\end{tabular}

$C R$ complete response, $P D$ progressive disease, $P R$ partial response, $S D$ stable disease

a Excluding three patients enrolled, but not treated

In the phase 3 study, 44 patients were randomized to CS-PHP and 49 to BAC [39]. The majority of patients $(81.6 \%)$ in the BAC group received active treatment (most frequently with temozolamide) whereas $18.4 \%$ of patients received only supportive care. A clinically meaningful and statistically significant improvement in hPFS was observed in the CS-PHP group compared to the BAC group. Median hPFS was 1.64 months (95\% CI 1.48-2.92) in the BAC group compared to 7.03 months (95\% CI 5.22-9.66) in the CS-PHP group $(P<0.001)$. Additionally, there was a statistically significant $\quad(P<0.0001)$ improvement in the hOR rate with PHP (36.4\%) compared to BAC (2.0\%). Median OS was similar in the CS-PHP and BAC groups; however, these results are confounded by the high number of BAC patients (57.1\%) who crossed over to CS-PHP treatment. Of the 49 patients originally assigned to BAC, 28 had hepatic disease progression and crossed over to receive CS-PHP. A post hoc analysis examined outcomes in these patients compared to those in the BAC group who had not crossed over to CS-PHP and those originally assigned to CS-PHP [40]. The baseline characteristics of the crossover group were comparable to the groups who received BAC only and those who were initially randomized to CS-PHP [39]. The analysis of outcomes in this post hoc analysis showed that the efficacy of CS-PHP in the 
crossover group was similar to that in the group initially randomized to this therapy. The median hPFS was 8.0 in the CS-PHP group and 1.6 in the BAC group that had not crossed over to CS-PHP $(P<0.0001)$. The crossover group had an hPFS of 8.8 months, which was comparable to that in the group initially treated with CS-PHP. The median OS was 9.8 months in the CS-PHP group as opposed to 4.1 months in the BAC-only group and 15.3 months in the crossover group. As was seen in the phase 2 study, the toxicity profile in the phase 3 study was characterized by adverse events related to bone marrow suppression, including thrombocytopenia, neutropenia, and anemia. Most of these events were not associated with clinical sequelae.

\section{Retrospective Studies}

In addition to the results of the clinical trial program, several retrospective studies reported outcomes data for CS-PHP with melphalan (summarized in Table 4). A total of four noncomparative studies were conducted [41-44]. One of the noncomparative studies enrolled 13 patients [44], 10 of whom were also included in a separate comparative retrospective analysis [45]. In addition, two case reports were published [46, 47]. All of the studies reported hepatic response data; two of the studies also reported toxicity data $[41,43]$.

In the noncomparative studies of CS-PHP with melphalan for the treatment of unresectable hepatic metastases, patients had a variety of primary tumor types, including ocular melanoma, cutaneous melanoma, CRC, leiomyosarcoma, breast cancer, cholangiocarcinoma, and gastric cancer (Table 4) [41-44]. Among the 54 patients in these four studies, hepatic response rates by RECIST criteria were 58\%, 75\%, 70\%, and
$61.5 \%$, respectively. Hepatic responses were seen in patients with cholangiocarcinoma, CRC, ocular melanoma, and cutaneous melanoma, with complete responses in one patient with cholangiocarcinoma and two patients with ocular melanoma. Stable disease was also observed with CS-PHP in patients with ocular melanoma, cutaneous melanoma, melanoma with unknown primary, leiomyosarcoma, breast cancer, and gastric cancer.

Toxicity data were reported in the noncomparative study by Vogl et al. [41]. Toxicities following CS-PHP were consistent with the toxicity profile seen in the clinical trials of CS-PHP with melphalan. The most common adverse effects were hematologic events (thrombocytopenia, anemia, neutropenia), which were managed effectively with supportive measures. Three patients were initially treated with the first-generation filter, then switched to the second-generation filter, providing an opportunity for a comparison of toxicity profiles with the two systems. Toxicity was less severe and patients required fewer supportive measures (i.e., no transfusions, shorter courses of colony stimulating factors) with the second-generation filter than the first-generation filter.

The comparative study was a retrospective evaluation of outcomes at a single institution in a small number of patients $(N=30)$ with unresectable hepatic metastases from ocular melanoma, cutaneous melanoma, or an unknown primary tumor who received a liver-directed therapy between 2008 and 2014 (Table 4) [37]. Liver-directed therapies included yttrium, chemoembolization, and CS-PHP. One patient was treated with yttrium after CS-PHP and one patient was treated with CS-PHP after chemoembolization; both of these patients were excluded from the efficacy analyses. 


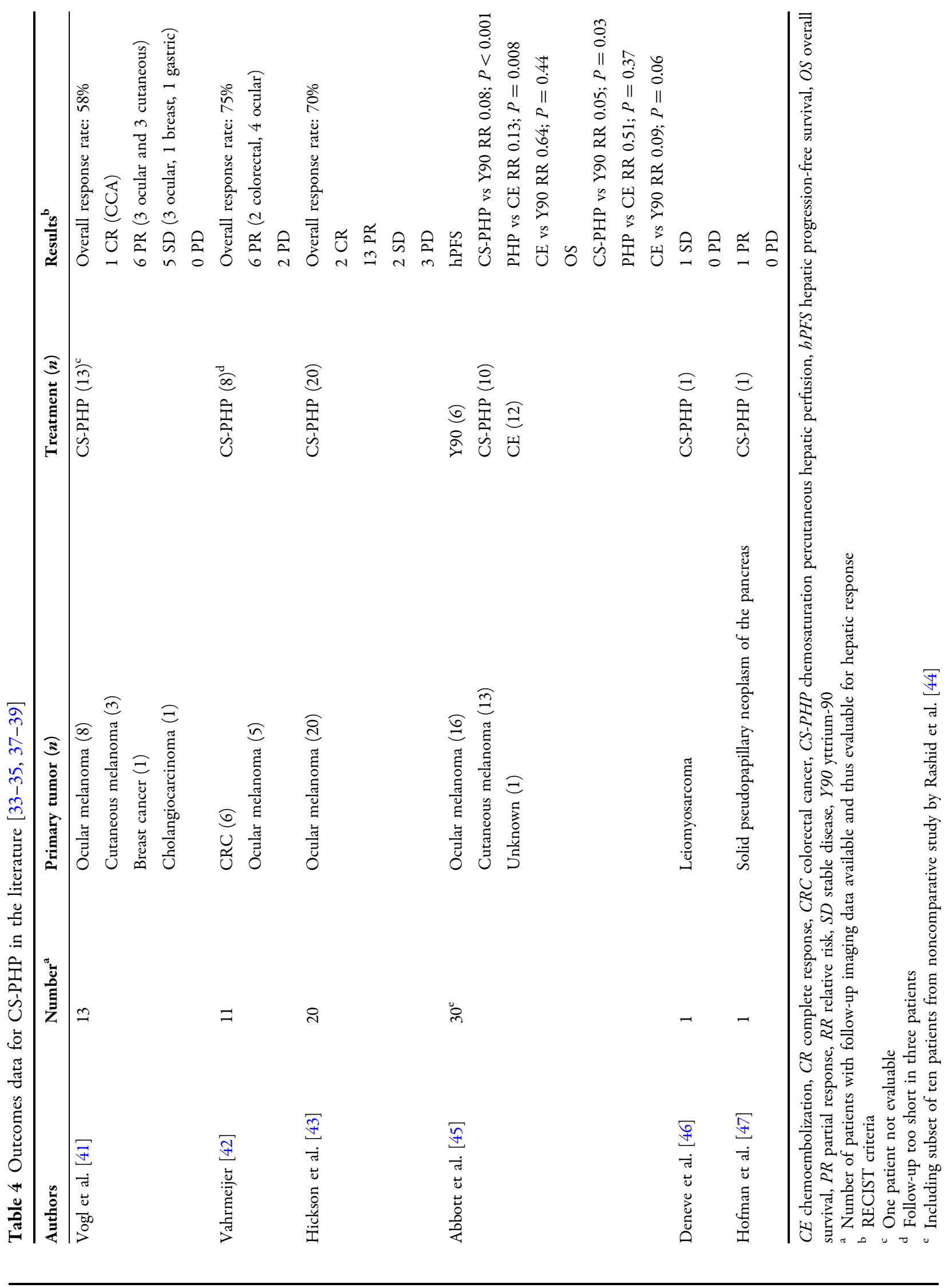


Kaplan-Meier survival estimates, log-rank test, and multivariate Cox regression analyses were used to relate patient, tumor, and treatment variables to hPFS and OS.

There were no differences between the yttrium, chemoembolization, and CS-PHP groups with regard to age, adjuvant therapy, prior regional hepatic treatment, or complications following treatment. Extrahepatic disease was more prevalent in the chemoembolization group $(P=0.004)$ compared with the yttrium and CS-PHP groups. The ECOG score trended lower in the CS-PHP group $(P=0.051)$ compared with the yttrium and chemoembolization groups.

Median hPFS was significantly $(P=0.002)$ longer with CS-PHP (310 days) than with yttrium (54 days) and chemoembolization (80 days) [36]. Median hPFS was also significantly longer with CS-PHP versus yttrium $(P<0.001)$ and CS-PHP versus chemoembolization $(P=0.008)$, but not yttrium versus chemoembolization $(P=0.44)$. A higher ECOG score $(P=0.01)$ and a greater tumor burden $(P=0.03)$ were correlated with a shorter duration of hPFS. Median OS was longer with CS-PHP (736 days) than with yttrium (285 days) and chemoembolization (265 days); this difference was significant for CS-PHP versus yttrium $(P=0.03)$. Neither ECOG score nor tumor burden were significant predictors of OS.

\section{Case Reports}

Two case reports for CS-PHP with melphalan have been published (Table 4) [46, 47]. Hofman et al. reported the case of a 40-year-old woman with unresectable hepatic metastases from a solid pseudopapillary neoplasm of the pancreas who had progressive liver disease after 3 months of systemic chemotherapy (gemcitabine and cisplatin) [47]. CS-PHP was performed twice within 8 weeks and a partial response was observed. The patient recovered rapidly after each CS-PHP procedure. Grade 3 leukopenia was seen after the second procedure and was effectively managed with granulocyte colony stimulating factor. No other toxicities were evident.

Deneve et al. described the case of a 66-year-old woman with isolated bilobar unresectable hepatic metastases from leiomyosarcoma [46]. Four target hepatic lesions were identified and monitored to assess treatment response. The patient underwent four CS-PHP procedures with melphalan. A 25\% reduction in the size of the largest target lesion was observed, and the hPFS was 16 months. With the first CS-PHP procedure, the patient had mild bone marrow suppression, including thrombocytopenia and neutropenia, which were managed with filgrastim and platelet transfusion, respectively. Minimal toxicity and a more rapid recovery were seen with the second CS-PHP procedure.

\section{Post-approval Experience with CS-PHP in Europe}

As of July 31, 2016, 186 patients in the EU had received a total of 321 CS-PHP treatments (Table 5). Most procedures were performed for patients with liver metastases from ocular melanoma. The majority of procedures were performed in Germany (75 procedures total in 11 hospitals), the UK (49 procedures total in four hospitals), and the Netherlands (33 procedures total in two hospitals). CS-PHP was also performed in Italy (12 procedures total in two hospitals), France (nine procedures total in two hospitals), Spain (six procedures total in two hospitals), Ireland (one procedure total in one hospital), and Turkey (one procedure in one hospital). 
Table 5 Number of CS-PHP treatments in Europe by tumour type

\begin{tabular}{lc}
\hline Tumor type & CS-PHP treatments $(\boldsymbol{N})$ \\
\hline Ocular melanoma & 213 \\
Cutaneous melanoma & 9 \\
Cholangiocarcinoma & 41 \\
Breast cancer & 5 \\
Hepatocellular carcinoma & 13 \\
NET & 4 \\
Anal mucosal melanoma & 1 \\
Pancreatic cancer & 11 \\
Colorectal cancer & 20 \\
Sarcoma & 1 \\
Gastric cancer & 1 \\
Endometrium cancer & 1 \\
Prostate & 1 \\
Total & 321 \\
\hline CRC colorectal cancer, & $C S-P H P$ chemosaturation \\
percutaneous hepatic perfusion, $H C C$ hepatocellular \\
carcinoma, NET neuroendocrine tumor
\end{tabular}

An EU registry has been initiated to collect safety, efficacy, and resource utilization data for patients who have received CS-PHP for treatment of an unresectable hepatic malignancy. Delcath is contacting its list of authorized customer hospitals that have been supplied the Hepatic CHEMOSAT ${ }^{\circledR}$ Delivery System to request that they identify patients who have received or who will be receiving CS-PHP treatment for inclusion in this registry. Efficacy data that will be collected include hepatic response to treatment; time to partial response or complete response; time to liver metastasis progression; time to extrahepatic disease progression, and overall survival. Safety data that will be collected include laboratory test results; length of postoperative stay; blood product use; hospital admission and reason for admission within 30 days following CS-PHP; disease- and treatment-related adverse events; supportive care (i.e., growth factors and antibiotics); performance status; tumor-related symptoms; and quality of life. The registry will end when data for 200 patients have been accrued.

\section{Ongoing Clinical Studies}

A number of clinical studies with CS-PHP are either ongoing or planned. A summary of these studies is provided in Table 6 .

\section{CONCLUSIONS}

CS-PHP with melphalan is an effective regional treatment option for patients with unresectable primary or hepatic metastases. The toxicities associated with CS-PHP are in most cases transient and manageable. In the clinical trial program (specifically, the randomized, controlled phase 3 study), CS-PHP with melphalan resulted in a clinically meaningful and statistically significant improvement in hPFS in patients with unresectable hepatic metastases from ocular or cutaneous melanoma. Clinically meaningful hepatic responses in patients with hepatocellular carcinoma or NET were also observed in the clinical trial program. The safety profile of CS-PHP with melphalan is well characterized, consisting primarily of bone marrow suppression (i.e., neutropenia, anemia, and thrombocytopenia). These toxicities can be addressed by a combination of patient selection criteria, patient monitoring, and appropriate intervention.

In addition, results from a number of published studies and case reports indicate that CS-PHP with melphalan may also be a 
Table 6 Ongoing or planned clinical studies with CS-PHP

\begin{tabular}{|c|c|c|}
\hline Trial & Tumor type & Objectives \\
\hline \multicolumn{3}{|l|}{ Phase II studies } \\
\hline \multirow[t]{4}{*}{$\begin{array}{l}\text { Study } 201(N=11) \\
\text { (USA only) }\end{array}$} & \multirow[t]{4}{*}{$\begin{array}{l}\text { Unresectable HCC (confined to } \\
\text { the liver) }\end{array}$} & $\begin{array}{l}\text { Safety and efficacy of CS-PHP followed by sorafenib, } \\
\text { including the following: }\end{array}$ \\
\hline & & Evaluate hORR, PFS, and safety \\
\hline & & Characterize systemic exposure of melphalan \\
\hline & & Assess patient quality of life \\
\hline \multirow[t]{5}{*}{$\begin{array}{l}\text { Study } 202(N=11) \\
\text { (EU only) }\end{array}$} & \multirow[t]{4}{*}{$\begin{array}{l}\text { Unresectable HCC (confined to } \\
\text { the liver) }\end{array}$} & $\begin{array}{l}\text { Safety and efficacy of CS-PHP without sorafenib, including } \\
\text { the following: }\end{array}$ \\
\hline & & Evaluate hORR, PFS, and safety \\
\hline & & Characterize systemic exposure of melphalan \\
\hline & & Assess patient quality of life \\
\hline & $\begin{array}{l}\text { Unresectable ICC (confined to } \\
\text { the liver) }\end{array}$ & Cohort to be added to Study 202 \\
\hline \multicolumn{3}{|l|}{ Phase III studies } \\
\hline $\begin{array}{l}\text { Planned } \\
N=240\end{array}$ & $\begin{array}{l}\text { Ocular melanoma with liver } \\
\text { metastases }\end{array}$ & \multirow[t]{2}{*}{ Global study to start in 2016; primary endpoint, OS } \\
\hline $\begin{array}{l}\text { Investigator- initiated } \\
\text { studies }\end{array}$ & Metastatic CRC & \\
\hline \multicolumn{3}{|l|}{$\begin{array}{l}\text { University of Leiden, } \\
\text { the Netherlands }\end{array}$} \\
\hline$N=31$ & & \\
\hline
\end{tabular}

CRC colorectal cancer, CS-PHP chemosaturation percutaneous hepatic perfusion, $H C C$ hepatocellular carcinoma, bORR hepatic objective response rate, $h P F S$ hepatic progression-free survival, ICC intrahepatic cholangiocarcinoma, $O S$ overall survival, $P F S$ progression-free survival

therapeutic option for other primary and secondary liver tumors providing the rationale for ongoing and planned clinical trials across a spectrum of tumor histologies including ocular or cutaneous melanoma, CRC, and cholangiocarcinoma.

\section{ACKNOWLEDGEMENTS}

All named authors meet the International Committee of Medical Journal Editors (ICMJE) criteria for authorship for this manuscript, take responsibility for the integrity of the work as a whole, and have given final approval to the version to be published. Prof. Arndt Vogel acts as the guarantor for this paper. This review, the article processing charges, and the open access fee were funded by Delcath Systems Inc., New York, NY, USA. In addition to the authors, the following European experts in CS-PHP contributed to the development of this article: Stephen Fenwisk, Aintree, Liverpool, 
UK; Harald Keller, Universitätsklinikum Frankfurt am Main, Germany; and Dr. Pradesh Kumar, Aintree, Liverpool, UK. Editorial assistance in the preparation of this manuscript was provided by Nancy Baxter of Advyzom. Support for this assistance was funded by Delcath Systems.

Disclosures. A. Vogel is an advisor and has received a speaker honorarium from Delcath Systems. S. Gupta is a proctor anesthesiologist receiving financial remuneration on a case-by-case basis from Delcath Systems and is an advisor anesthesiologist, receiving no compensation, for Delcath Systems. M. Zelle is a proctor for Delcath Systems and a consultant for Boston Scientific. R. von Haken has received honoraria from Orionpharma, Merck Sharpe \& Dohme, and Köhler Chemie and is an advisor for Orionpharma. G. Lotz is a proctor anesthesiologist receiving financial renumeration on a case-by-case basis from Delcath Systems. T. Vogl has received speaker honoraria and travel stipends from Delcath Systems, honoraria for serving on an advisory board for Siemens, and travel stipends from Amica, Covidien, General Electric, and Pharmacept. Frank Wacker has received a research grant from Delcath Systems and financial remuneration from Promedicus Ltd. and Siemens Healthcare. R. Brüning and A. Vahrmeijer have nothing to disclose.

Compliance with Ethics Guidelines. This article does not contain any new studies with human or animal subjects performed by any of the authors.

Data availability. Data sharing is not applicable to this article as no new datasets were generated or analyzed during the current study.
Open Access. This article is distributed under the terms of the Creative Commons Attribution-NonCommercial 4.0 International License (http://creativecommons.org/licenses/ by-nc/4.0/), which permits any noncommercial use, distribution, and reproduction in any medium, provided you give appropriate credit to the original author(s) and the source, provide a link to the Creative Commons license, and indicate if changes were made.

\section{REFERENCES}

1. Llovet JM, Ricci S, Mazzaferro V, et al. Sorafenib in advanced hepatocellular carcinoma. N Engl J Med. 2008;359:378-90.

2. Wang C, Wang H, Yang W, et al. Multicenter randomized controlled trial of percutaneous cryoablation in hepatocellular carcinoma. Hepatology. 2015;61:1579-90.

3. Yamada R, Sato M, Kawabata M, et al. Hepatic artery embolization in 120 patients with unresectable hepatoma. Radiology. 1983;148:397-401.

4. Breedis C, Young G. The blood supply of neoplasms in the liver. Am J Pathol. 1954;30:969-77.

5. Raoul JL, Heresbach D, Bretagne JF, et al. Chemoembolisation of hepatocellular carcinomas. A study of the biodistribution and pharmacokinetics of doxorubicin. Cancer. 1992;70:585-90.

6. Hong K, Khwaja A, Liapi E, et al. New intra-arterial drug delivery system for the treatment of liver cancer: preclinical assessment in a rabbit model of liver cancer. Clin Cancer Res. 2006;12:2563-7.

7. Kettenbach J, Stadler A, Karzler IV, et al. Drug-loaded microspheres for the treatment of liver cancer: review of current results. Cardiovasc Interv Radiol. 2008;31:468-76.

8. Valpione S, Aliberti C, Parrozzani R, et al. A retrospective analysis of 141 patients with liver metastases from uveal melanoma: a two-cohort study comparing transarterial chemoembolization with CPT-11 charged microbeads and historical treatments. Melanoma Res. 2015;25:164-8.

9. Abdelmaksoud MH, Louie D, Hwang GL, et al. Yttrium-90 radioembolization of renal cell 
carcinoma metastatic to the liver. J Vasc Interv Radiol. 2012;23:323-30.

10. Alexander HR, Libutti SK, Bartlett DL, et al. A phase I-II study of isolated hepatic perfusion using melphalan with or without tumour necrosis factor for patients with ocular melanoma metastatic to liver. Clin Cancer Res. 2000;6:3062-70.

11. Alexander HR, Libutti SK, Pingpank JF, et al. Hyperthermic isolated hepatic perfusion using melphalan for patients with ocular melanoma metastatic to liver. Clin Cancer Res. 2003;9:6343-9.

12. Noter SL, Rothbaerth J, Pijl ME, et al. Isolated hepatic perfusion with high-dose melphalan for the treatment of uveal melanoma metastases confined to the liver. Melanoma Res. 2004;14:67-72.

13. Rizell M, Mattson J, Cahlin C, et al. Isolated hepatic perfusion for liver metastases of malignant melanoma. Melanoma Res. 2008;18:120-6.

14. Feldman ED, Wu PC, Beresneva T, et al. Treatment of patients with unresectable primary hepatic malignancies using hyperthermic isolated hepatic perfusion. J Gastrointest Surg. 2004;8:200-7.

15. van Iersel LB, Hoekman EJ, Gelderblom H, et al. Isolated hepatic perfusion with $200 \mathrm{mg}$ melphalan for advanced noncolorectal liver metastases. Ann Surg Oncol. 2008;15:1891-8.

16. Tanis E, Nordlinger $B$, Mauer $M$, et al. Local recurrence rates after radiofrequency ablation or resection of colorectal liver metastases. Analysis of the European Organisation for Research and Treatment of Cancer \#40004 and \#40983. Eur J Cancer. 2014;50:912-9.

17. Wasan HS. Selective internal radiation therapy first-line therapy-SIRFLOX and FOXFIRE. Eur J Cancer Suppl. 2012;10(3):12-4.

18. Ruers T, Punt CJA, van Coevorden $\mathrm{R}$, et al. Radiofrequency ablation (RFA) combined with chemotherapy for unresectable colorectal liver metastases (CRC LM): long-term survival results of a randomized phase II study of the EORTC-NCRI cCSG-ALM Intergroup 40004 (CLOCC). J Clin Oncol. 2015;33(suppl):Abstr 3501.

19. Ku Y, Iwasaki TT, Tominaga $\mathrm{M}$, et al. Reductive surgery plus percutaneous isolated hepatic perfusion for multiple advanced hepatocellular carcinoma. Ann Surg. 2004;239(1):53-60.

20. International Agency for Research on Cancer. http://www.iarc.fr/. Accessed 1 Jan 2016.

21. Bruix J, Sherman M, American Association for the Study of Liver Disease. Management of hepatocellular carcinoma: an update. Hepatology. 2011;53(3):1020-2.

22. Llovet JM, Real MI, Montana X, et al. Arterial embolization or chemoembolization versus symptomatic treatment in patients with unresectable hepatocellular carcinoma: a randomised controlled trial. Lancet. 2002;359(9319):1734-9.

23. Lo CM, Ngan H, Tso WK, et al. Randomized controlled trial of transarterial lipiodol chemoembolization for unresectable hepatocellular carcinoma. Hepatology. 2002;35(5):1164-71.

24. Macias RIR. Cholangiocarcinoma: biology, clinical management, and pharmacological perspectives. ISRN Hepatol. 2014;2014:828074. doi:10.1155/ 2014/828074.

25. Horgan AM, Amir E, Walter T, Knox JJ. Adjuvant therapy in the treatment of biliary tract cancer: a systematic review and meta-analysis. J Clin Oncol. 2012;30(16):1934-40.

26. Valle JW, Furuse J, Jital M, et al. Cisplatin and gemcitabine for advanced biliary tract cancer: a meta-analysis of two randomized trials. Ann Oncol. 2014;25(2):391-8.

27. Ananthakrishnan A, Gogineni V, Saeian K. Epidemiology of primary and secondary liver cancers. Semin Int Radiol. 2006;23:47-63.

28. Egan KM, Seddon JM, Glyunn RJ, et al. Epidemiologic aspects of uveal melanoma. Surv Ophthalmol. 1988;32:239-51.

29. Norstein J, Silwn W. Natural history of liver metastases from colorectal carcinoma. J Gastrointest Surg. 1997;1:398-407.

30. Goldberg RM, Rothenberg ML, Van Custerm E, et al. The continuum of care: a paradigm for the management of metastatic colorectal cancer. Oncologist. 2007;12:38-50.

31. Chamberlain RS, Canes D, Brown KT, et al. Hepatic neuroendocrine metastases: does intervention alter outcomes? J Am Coll Surg. 2000;190:432-5.

32. Marinelli A, Vahrmeijer SL, van de Velde CJ. Phase I/II studies of isolated hepatic perfusion with mitomycin $\mathrm{C}$ or melphalan in patients with colorectal cancer hepatic metastases. Recent Results Cancer Res. 1998;147:83-94.

33. Vahrmeijer AL, van Dierendonck JH, Keizer HJ, et al. Increased local cytostatic drug exposure by isolated hepatic perfusion: a phase I clinical and pharmacologic evaluation of treatment with high 
dose melphalan in patients with colorectal cancer confined to the liver. Br J Cancer. 2000;82:1539-46.

34. de Vries MR, Borel Rinkes IH, van de Velde CJ, et al. Isolated hepatic perfusion with tumour necrosis factor alpha and melphalan: experimental studies in pigs and phase I data from humans. Recent Results Cancer Res. 1998;147:107-9.

35. van Iersel LB, Gelderblom H, Vahrmeijer AL, et al. Isolated hepatic melphalan perfusion of colorectal liver metastases: outcome and prognostic factors in 154 patients. Ann Oncol. 2008;19:1127-34.

36. van Iersel LB, de Leede EM, Vahrmeijer AL, et al. Isolated hepatic perfusion with oxaliplatin combined with $100 \mathrm{mg}$ melphalan in patients with metastases confined to the liver: a phase I study. Eur J Surg Oncol. 2014;40:1557-63.

37. Miao N, Pingpank JF, Alexander $H R$, et al. Percutaneous hepatic perfusion in patients with metastatic liver cancer: anesthetic, hemodynamic, and metabolic considerations. Ann Surg Oncol. 2008;15:815-23.

38. Pingpank JF, Libutti SK, Chang R, et al. Phase I study of hepatic arterial melphalan infusion and hepatic venous hemofiltration using percutaneously placed catheters in patients with unresectable hepatic malignancies. J Clin Oncol. 2005;23(15):3465-74.

39. Lillemoe HA, Alexander HR. Current status of percutaneous hepatic perfusion as regional treatment for patients with unresectable hepatic metastases: a review. Am Oncol Hematol Rev. 2014;15-23.

40. Alexander HR, Phase 3 Investigators. Hepatic perfusion $\left(\right.$ CHEMOSAT $^{\circledR}$ or CS-PHP) of melphalan vs. best alternative care (BAC) in patients with hepatic metastases from melanoma: update of a randomized phase 3 study. Ann Oncol. 2012;23(suppl 9):1141.

41. Vogl TJ, Zangos S, Scholtz JE, et al. Chemosaturation with percutaneous hepatic perfusions of melphalan for hepatic metastases: experience from two European centers. Rofo. 2014;186:937-44.

42. Vahrmeijer AL. Liver metastases: a new treatment paradigm. Presented at: 34th Congress of the European Society of Surgical Oncology; Liverpool, 2014; p. 29-31.

43. Hickson G, Karydis I, Wheater MJ, et al. Single centre experience of chemosaturation percutaneous hepatic perfusion in the treatment of metastatic uveal melanoma. J Clin Oncol. 2015;33(18 suppl):e20000.

44. Rashid OM, Choi J, Chaudhry T, et al. A single institution experience with percutaneous hepatic perfusion for unresectable melanoma or sarcoma to the liver. Presented at: 34th Congress of the European Society of Surgical Oncology; Liverpool, 2014; p. 29-31.

45. Abbott AM, Kim Y, Gandle OM, et al. Hepatic progression free survival and overall survival after regional therapy to the liver for metastatic melanoma. Ann Surg Oncol. 2015;22(2 suppl):Poster 255.

46. Deneve JL, Choi J, Gonzalez RJ, et al. Chemosaturation with percutaneous hepatic perfusion for unresectable isolated hepatic metastases from sarcoma. Cardiovas Interv Radiol. 2012;35:1480-7.

47. Hofmann $\mathrm{H}$, von Haken $\mathrm{R}$, Werner $\mathrm{J}$, et al. Unresectable isolated hepatic metastases from solid pseudopapillary neoplasm of the pancreas: a case report of chemosaturation with high-dose melphalan. Pancreatology. 2014;14:546-9. 